\section{Schizophrenia and mixed-handedness}

The new meta-analysis by Hirnstein \& Hugdahl $^{1}$ shows that schizophrenia is robustly associated with non-right-handedness and thus makes a strong case for genetic links between schizophrenia, brain lateralisation and handedness. The association was obtained meta-analytically for psychometrically assessed handedness, and it was even stronger when handedness was assessed behaviourally. Of interest, the available evidence also allowed the tentative conclusion that this association might foremost be driven by mixed-handedness, rather than left-handedness, thus suggesting that the strength of handedness, rather than its direction, might actually be linked to schizophrenia.

With regard to this possibility, we emphasise that assessment and classification reliability is a pervasive problem in handedness research. Studies in this field often treat handedness as a binary variable, comprising only right $v$. left preferences, and frequently merely use single-item measures, or arbitrary criteria for handedness classification, when multi-item inventories are used.

However, recent evidence ${ }^{2}$ shows that psychometrically assessed handedness is in fact taxonic and discrete - that is, a matter of qualitative, rather than quantitative, differences consisting of the taxa of right-, left- and mixed-handedness. Similarly, psychometrically assessed footedness, earedness, and eyedness all consist of these three taxa, which, together with handedness, might be explained by underlying sidedness, which also consists of the above three taxa. Further, handedness has been found to be a biased indicator of mixed-sidedness, which might be explained by ubiquitous external and societal pressures promoting right-hand preference among actual non-right-handers. ${ }^{3}$ It therefore appears that footedness, obviously being less influenced by such external pressures, is the most important predictor of sidedness. $^{2}$

Given these facts, it is interesting to note that the Hirnstein \& Hugdahl meta-analysis was still able to hint at mixed-handedness as the probable factor driving the association between schizophrenia and non-right-handedness, thereby making a convincing case for the power of the meta-analytical approach, which, through data aggregation, might overcome methodological problems of individual studies. In addition, increasing the classification reliability of handedness has recently aided in clarifying the hypothesised seasonal pattern of birth months among left-handed men, ${ }^{4}$ a hypothesis for which the available previous evidence had been highly inconsistent.

We thus recommend utilising psychometrically validated scales, along with a trichotomous classification, in investigations on schizophrenia and handedness, expecting that mixed-handedness might turn out to be more relevant than left-handedness for explaining this link. Further, we recommend intensifying research specifically with regard to footedness, which might be more important with regard to brain lateralisation than handedness, ${ }^{5}$ and might provide more direct, and thus more robust, behavioural evidence for the links between schizophrenia and brain lateralisation.

1 Hirnstein M, Hugdahl K. Excess of non-right-handedness in schizophrenia: meta-analysis of gender effects and potential biases in handedness assessment. Br J Psychiatry 2014; 205: 260-7.

2 Tran US, Stieger S, Voracek M. Evidence for general right-, mixed-, and leftsidedness in self-reported handedness, footedness, eyedness, and earedness, and a primacy of footedness in a large-sample latent variable analysis. Neuropsychologia 2014; 62: 220-32.

3 Porac C, Coren S. Lateral Preferences and Human Behavior. Springer, 1981.

4 Tran US, Stieger S, Voracek M. Latent variable analysis indicates that seasonal anisotropy accounts for the higher prevalence of left-handedness in men. Cortex 2014; 57: 188-97.

5 Elias LJ, Bryden MP. Footedness is a better predictor of language lateralisation than handedness. Laterality 1998; 3: 41-51.

Ulrich S. Tran, Martin Voracek, Department of Basic Psychological Research and Research Methods, School of Psychology, University of Vienna, Vienna, Austria. Email: ulrich.tran@univie.ac.at

doi: 10.1192/bjp.207.2.178 